\section{Concise cosmology}

\author{
The Little Book of the Big Bang: A \\ Cosmic Primer \\ by Craig Hogan \\ Copernicus: 1998. Pp. 181. \$20, 115.50

\section{George Ellis}

The explosion of data and theory in cosmology has led to a profusion of books about modern cosmology written for non-experts. Craig Hogan's cosmic primer ranks among the best: it is a gem. It is concisely written and very carefully crafted, with useful summary tables and diagrams. From a visual viewpoint it is restrained (there are no colour illustrations or photographs), but it provides a superb overview of cosmic processes (for example, nucleosynthesis and the origin of the cosmic background radiation spectrum). There are many interesting insights deriving from Hogan's deep involvement with the field; these will interest professionals as well as newcomers to cosmology.

Hogan raises and answers the main questions the uninitiated want answered: "Is everything expanding?", "What is the Universe expanding into?", "What happened before the Big Bang?", and so forth. He draws many excellent informative analogies, for example pointing out that the dynamical

equations of the expanding Universe are the same as those of a ball thrown vertically from the surface of the Earth, so the same set of possibilities arise in its solutions.

But there are weak points. An issue regarding the book's presentation is related to a fundamental dilemma in any course or text: whether to present the material in the order that is easiest to understand or in a more logical order that is more satisfying but harder to get to grips with. Hogan has chosen the logical approach. As a result, many readers who would appreciate the easily grasped later sections on the Big Bang which are accessible enough for high-school students and the general public - may well fall by the wayside during the second and third chapters; these contain an excellent but technically demanding summary of space and time scales and modern fundamental physics, for which a first-year university physics course is probably required. Readers who struggle with these should skip them at first reading and return to them later; ideally these chapters should be shifted to the end of the book.

Of broader concern is the degree of certainty with which various theories are presented. Hogan is generally careful about this, for example emphasizing that we cannot obtain evidence about events outside our past light cone (so discussions of the nature

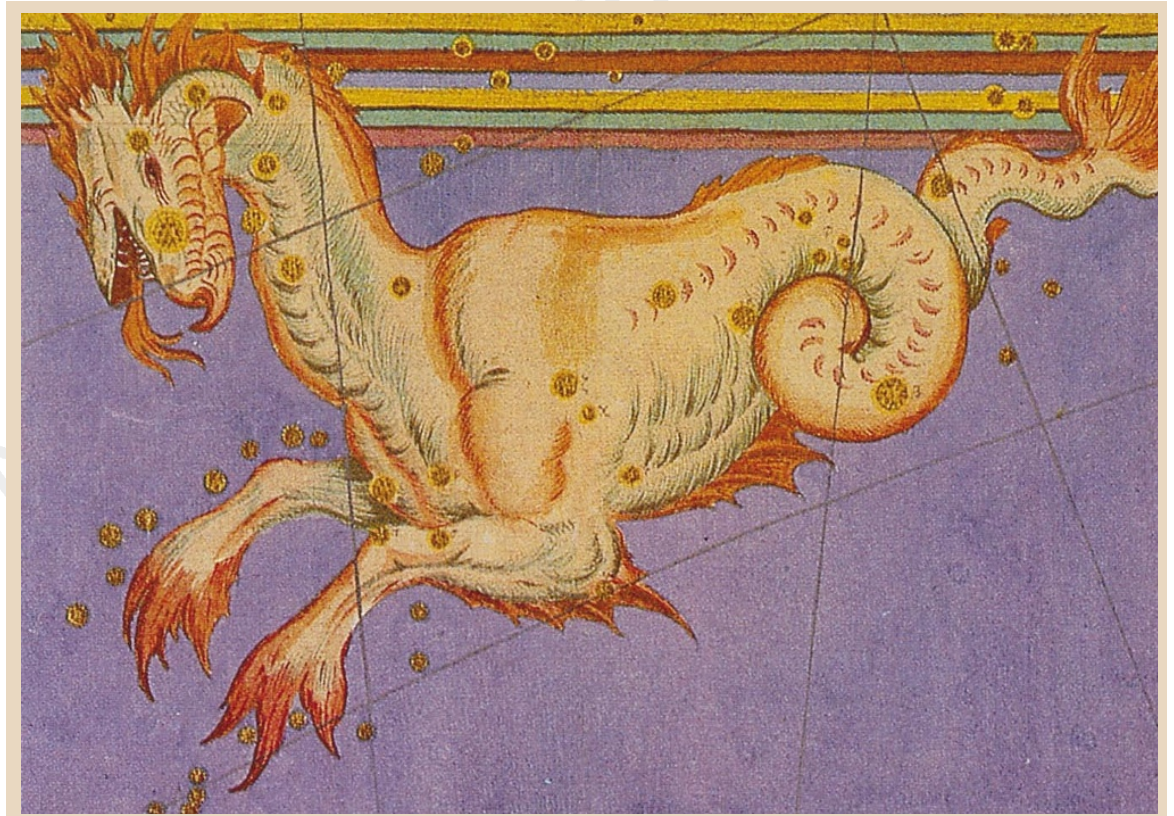

Publishing constellations

The constellation Cetus, from the sky atlas by Bayer (1603), as reproduced in Jay $M$. Pasachoff's The Peterson Field Guide to the Stars and Planets (Houghton Mifflin, \$18 (pbk)). It joins several other recent astronomical and cosmological primers, including Arthur Upgren's Night Has a Thousand Eyes: A NakedEye Guide to the Sky, Its Science, and Lore
(Plenum, \$27.95), T. Padmanabhan's After the First Three Minutes: The Story of Our Universe

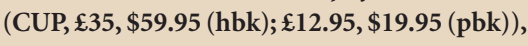
Marcelo Gleiser's The Dancing Universe: From Creation Myths to the Big Bang (Dutton, \$25.95) and Timothy Ferris's The Whole Shebang: A State-of-the-Universe(s) Report

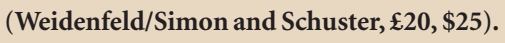

of the Universe on a super-Hubble scale are of a rather academic nature), but he restricts his discussion to a critical-density inflationary Universe, and in various places presents the inflationary Universe idea as a wellestablished theory. As he says himself, the relevant physics at times before the electroweak epoch is not well established; inflation is still a hypothesis rather than a fact. Furthermore, the density of matter in the universe is probably less than the critical value. A few more cautionary notes about this proposal would be in order.

A brief section at the end relates to the meaning of the Universe and anthropic issues. Although the technical side of this discussion is well done, any treatment of ideas of self and free will (as briefly mentioned here) requires a more extended and philosophically developed discussion. For example, Hogan states that "the most important thing about us is what we create: our perceptions and technologies, our culture and societies, our art and our science." This is a highly debatable philosophical position; many would claim in contrast that the most important thing about us is how we act, expressing our ethical stance and moral purpose. In discussing meaning-oflife issues, one cannot avoid such terrain.

There is a misleading diagram that represents our past light cone as like a conical hat, the apex being the present day, flaring out at the bottom towards the surface of last scattering. The ever-increasing size of the light cone as one goes back into the past is because the diagram represents the present-day distances of the objects concerned. But this is completely different from their distances when they emitted the light, so the actual shape of the past light cone is quite different from that shown. Its true shape is like an onion with the sharp edge upwards; it flares out to a maximum radius (at a redshift of 1.25 in a critical-density Universe) and then plunges sharply back in towards the hot early phase. In my view it would be much better to have that shape represented in this diagram. The figure does not show the maximum size of the past light cone in the past, nor the associated maxima of angular separation of null geodesics that result in angular sizes of rigid rods having a minimum.

A second technical issue is the claim that, in a Universe that lasts forever, despite infinite time being available, there would not be enough time to think all possible thoughts. This conclusion is based on allowing the coding of these thoughts to require an infinite chain of symbols, but that does not make sense. It must be possible to code any thought that is meaningful in a finite number of symbols, for otherwise it cannot be processed (we can think about infinity, for example, precisely because it can be coded in a single symbol). There are effectively 
only a (very large) finite number of possible distinct brain states in any brain of finite size.

These comments do not detract from the overall value of the presentation, which I warmly recommend. I am sure there will be many further editions of this fine book. $\square$ George Ellis is in the Mathematics Department, University of Cape Town, Rondebosch 7700, Cape Town, South Africa.

\section{Sex and sensibility}

\section{The Two Sexes: Growing Up Apart, Coming Together \\ by Eleanor E. Maccoby \\ Harvard University Press: 1998. Pp. 376. $\$ 39.95, £ 26.50$}

\section{David H.Skuse}

Are men really from Mars and women from Venus? In The Two Sexes, Eleanor E. Maccoby attempts to explain the considerable evidence that male and female patterns of social interaction differ from one another in terms of both same-sex and opposite-sex exchanges.

In 1974, Maccoby published with Carol Jacklin a landmark work on the psychology of sex differences, which arguably heralded the birth of a movement in feminist psychology. Stimulated by the conclusions of that synthesis of previous research, which claimed such differences were smaller than had been generally assumed, feminist scholars set out to show that, if gender studies were conducted with due regard to cultural relativity and gender stereotyping (by subject and observer), consistent findings would emerge. They reasoned that the results would show few, small or no differences in cognitive and social characteristics by sex.

Many meta-analyses later, only the most diehard psychologist would still cling to the view that sex differences in behaviour do not exist. Meanwhile, Maccoby has become a proponent of the popular 'separate cultures' school, which argues that children learn rules for social interaction from experience in largely peer-aggregated groups. They carry this learning through to adulthood. So when a man's wife asks him "What's wrong, honey?", and he replies "Nothing", and she persists "I can tell you're annoyed with me - I can see it in your face", he retorts "I tell you, there's nothing wrong, cut it out won't you!" because he is male, and that is how he has learned to respond to questions about his emotional state.

Maccoby's book is beautifully written and organized. In the first half she takes us on a journey from infancy through preschool behaviour, to elementary school and adolescence. Fascinating evidence is adduced for the formation of sex-segregated social groups, which are amazingly resistant

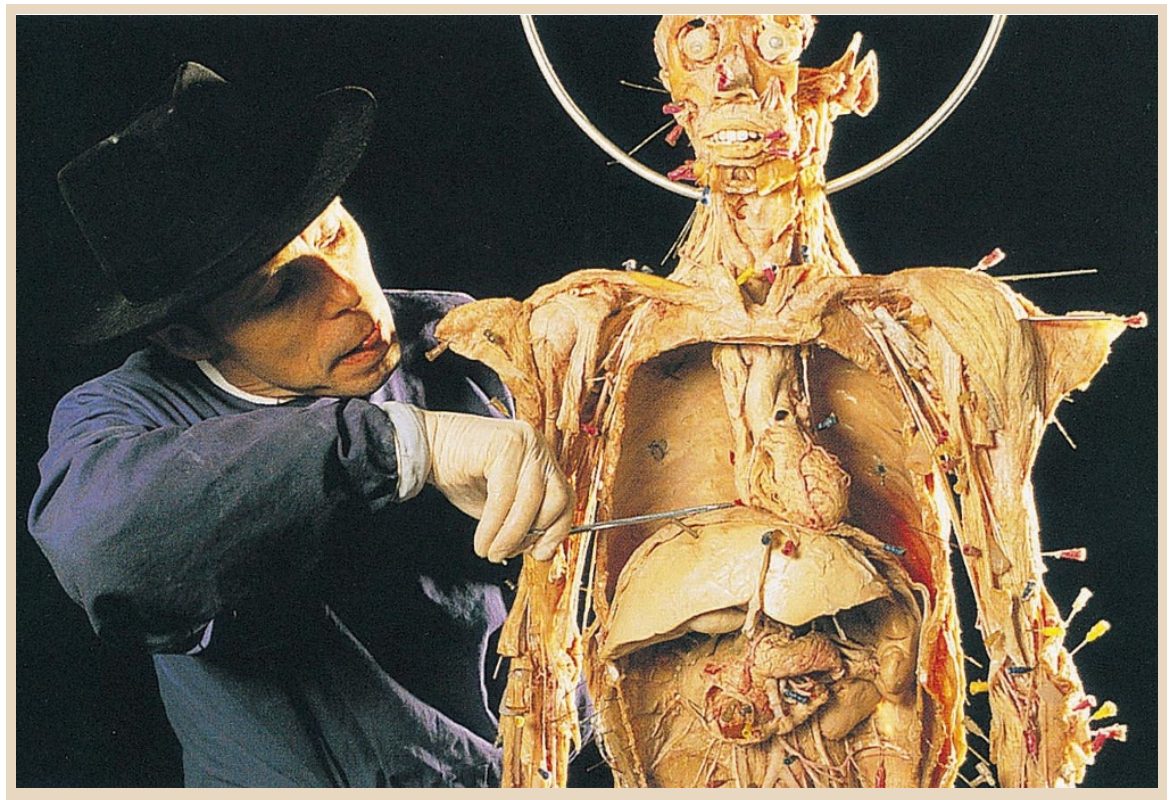

\section{Insides out}

The German anatomist Gunther von Hagens preserves human bodies by a process he calls 'plastination', in which he replaces all of the water and fat with silicone and other polymers. He began by creating anatomical preparations for educational purposes, but now also exhibits them at controversial shows. The corpses are obtained through a donation programme.

in middle childhood to well-meaning adult attempts to force integration. In the second half, which is rather weaker in terms of argument and grasp of detail, issues of sex roles in the workplace and in parenthood are discussed. A variety of explanations for the seemingly universal existence of sexrole differentiation are proposed, from biology to evolutionary psychology, but Maccoby is most comfortable and knowledgeable when discussing the role of social influences.

As writers on the subject acknowledge, despite a tremendous and growing literature there has been no really satisfactory theoretical framework to encompass both the intrinsic differences in the ways the sexes behave and the environmental influences that moderate or mediate such traits. Although evolutionary psychology claims the high ground here, many commentators feel that retrospective predictions fail to do justice to the complexity of the issues.

Take the well-documented male propensity to dominate not only his own sex but particular females too. How could this be linked to other typically male characteristics, such as a lack of emotional expression (relative to females) and the irritating habit of interrupting people (up to five times as often as women in small-group interactions)? Do these features of 'male' behaviour arise innately, from 'essential qualities',
This image by Marc Steinmetz of von Hagens at work is from the science and technology section of 1998 World Press Photo (Thames and Hudson, $\mathfrak{E} 12.95$ (pbk)), a remarkable collection of the previous year's most striking examples of photojournalism. The book brings together the winners of the 41 st World Press Photo Contest.

or are they by-products of socialization experiences?

Maccoby is rather surer about the origins of male behaviour than female behaviour. Gender divergence, she argues, has its origins in the way children of a given sex aggregate with children of the same age. Groups of male children tend to be more cohesive than female aggregations, and their play style (which she characterizes as rough and essentially physical in nature) is highly attractive to boys but is a turn-off to most girls. On the other hand, female groups are loose-knit affairs, although they are also formed on the basis of play-style compatibility and are, as any male will tell you, not at all attractive to boys. It is implicit that girls form groups with girls largely because they do not, as a rule, want to join in with the boys.

Could it be that boys are primed by androgen exposure in utero to behave in a way that fosters group cohesion? Maccoby seems happy to posit biological factors as the ultimate explanation for male social behaviour, but for girls an environmental explanation is proposed. She thinks that girls are influenced by their upbringing to be more sensitive to others' feelings and to be more nurturing, socially sensitive, friendly and concerned with another's welfare.

Maccoby believes the differentiation of social behaviour by sex in adulthood 